\title{
Witt groups of the punctured spectrum of a 3-dimensional regular local ring and a purity theorem
}

\author{
M. Ojanguren, R. Parimala, R. Sridharan and V. Suresh
}

\section{Introduction}

Let $A$ be a regular local ring with quotient field $K$. Assume that 2 is invertible in $A$. Let $W(A) \rightarrow W(K)$ be the homomorphism induced by the inclusion $A \hookrightarrow K$, where $W($ ) denotes the Witt group of quadratic forms. If $\operatorname{dim} A \leq 4$, it is known that this map is injective $([\mathbf{6}],[\mathbf{7}])$. A natural question is to characterise the image of $W(A)$ in $W(K)$. Let $\operatorname{Spec}^{1}(A)$ be the set of prime ideals of $A$ of height 1 . For $P \in \operatorname{Spec}^{1}(A)$, let $\pi_{P}$ be a parameter of the discrete valuation ring $A_{P}$ and $k(P)=A_{P} / P A_{P}$. For this choice of a parameter $\pi_{P}$, one has the second residue homomorphism $\partial_{P}: W(K) \rightarrow W(k(P))([\mathbf{9}]$, p. 209). Though the homomorphism $\partial_{P}$ depends on the choice of the parameter $\pi_{P}$, its kernel and cokernel do not. We have a homomorphism

$$
\partial=\left(\partial_{P}\right): W(K) \rightarrow \bigoplus_{P \in \operatorname{Spec}^{1}(A)} W(k(P))
$$

A part of the so-called Gersten conjecture is the following question on "purity":

Is the sequence

$$
W(A) \rightarrow W(K) \stackrel{\partial}{\rightarrow} \bigoplus_{P \in \operatorname{Spec}^{1}(A)} W(k(P))
$$

exact?

The above question has an affirmative answer for $\operatorname{dim}(A) \leq 2$ ([3], p.277, 
[1]). There have been speculations by Pardon and Barge-Sansuc-Vogel on the question of purity. However, in the literature, there is no proof for purity even for $\operatorname{dim}(A)=3$. One of the consequences of the main result of this paper is an affirmative answer to the purity question for $\operatorname{dim}(A)=3$.

We briefly outline our main result. For any scheme $X$ let $W^{\epsilon}(X)$ denote the Witt group of $\epsilon$-symmetric spaces on $X, \epsilon= \pm 1\left(W^{+1}(X)=W(X)\right.$ being the usual Witt group of symmetric spaces over $X)$. Let $A$ be a regular local ring of dimension 3 with maximal ideal $m$ and $Y=\operatorname{Spec}(A) \backslash\{m\}$. We associate $(\S 2)$ to an $\epsilon$-symmetric space over $Y$ a $(-\epsilon)$-symmetric space over a finite length $A$-module. This assignment leads to a homomorphism $W^{\epsilon}(Y) \rightarrow W_{f l}^{-\epsilon}(A)$, where $W_{f l}^{\epsilon}(A)$ is the Witt group of $\epsilon$-symmetric spaces of finite length $A$-modules $(\operatorname{cf} \S 0)$. Then we prove $(\S 3)$ that the sequence

$$
0 \rightarrow W^{\epsilon}(A) \rightarrow W^{\epsilon}(Y) \rightarrow W_{f l}^{-\epsilon}(A) \rightarrow 0
$$

is exact, where the map $W^{\epsilon}(A) \rightarrow W^{\epsilon}(Y)$ is induced by the restriction. Since $W_{f l}^{\epsilon}(A) \simeq W^{\epsilon}(A / m)$, it follows that $W_{f l}^{-1}(A)=0$. Thus the map $W(A) \rightarrow W(Y)$ is an isomorphism. This leads to the purity theorem for the Witt groups. On the other hand, since every skew-symmetric space over $A$ is hyperbolic, $W^{-1}(A)=0$ and we get an isomorphism $W^{-1}(Y) \simeq W(A / m)$. We observe the curious fact that if $A$ is complete, $W^{ \pm 1}(Y)$ is isomorphic to $W(A / m)$.

A crucial result used in our proof of the main theorem is a theorem of Horrocks $([\mathbf{2}])$ on vector bundles on the punctured spectrum $Y=\operatorname{Spec}(A) \backslash$ $\{m\}$, where $A$ is a regular local ring of dimension 3 and $m$ its maximal ideal. We use his theorem on the equivalence of the category of " $\Phi$-equivalence" classes of vector bundles on $Y$ with the category of finite length $A$-modules.

We would like to remark parenthetically that purity for dimension 3 was used in [8] while establishing the equivalence of finite generation of Witt groups of affine real 3 -folds and the finite generation of Chow groups of codimension 2 cycles modulo 2.

We thank the referee for patiently pointing out errors, obscurities and misprints in the various versions of this paper.

\section{0. $\epsilon$-symmetric spaces reminisced}

Let $A$ be a regular local ring of dimension three in which 2 is invertible. We recall the definition of $\epsilon$-symmetric spaces on finite length $A$-modules 
and their Witt groups. For $A$-modules $M, N$ and $i \geq 0$, let $\operatorname{Ext}^{i}(M, N)$ denote the group of congruence classes of $i$-fold extensions of $N$ by $M$ ([4], p. 84). For any homomorphism $f: M \rightarrow M^{\prime}$ of $A$-modules, let $\operatorname{Ext}^{i}(N, f)$ : $\operatorname{Ext}^{i}(N, M) \rightarrow \operatorname{Ext}^{i}\left(N, M^{\prime}\right)$ be the induced homomorphisms defined as follows: Let

$$
\zeta=0 \rightarrow M \stackrel{\alpha}{\rightarrow} Z_{i} \stackrel{\partial_{i}}{\rightarrow} Z_{i-1} \rightarrow \cdots \rightarrow Z_{2} \stackrel{\partial_{2}}{\rightarrow} Z_{1} \stackrel{\beta}{\rightarrow} N \rightarrow 0
$$

be an $i$-fold extension of $N$ by $M$. Let $Z=\left(Z_{i} \oplus M^{\prime}\right) /(\{(\alpha(x), f(x)) \mid x \in$ $M\})$ be the push-out of the diagram $([4])$

$$
\begin{array}{lll}
M & \stackrel{\alpha}{\rightarrow} & Z_{i} \\
\downarrow f & & \\
M^{\prime} & &
\end{array}
$$

Then

$$
\operatorname{Ext}^{i}(N, f)(\zeta)=0 \rightarrow M^{\prime} \stackrel{\alpha^{\prime}}{\rightarrow} Z \stackrel{\partial^{\prime}}{\rightarrow} Z_{i-1} \stackrel{\partial_{i-1}}{\rightarrow} \cdots \rightarrow Z_{2} \stackrel{\partial_{2}}{\rightarrow} Z_{1} \stackrel{\beta}{\rightarrow} N \rightarrow 0
$$

where $\alpha^{\prime}$ and $\partial^{\prime}$ are the natural homomorphisms induced by the push-out. Similarly, we define $\operatorname{Ext}^{i}(f, N)$ as the pull-back under $f$ of an $i$-fold extension of $N$ by $M^{\prime}$. Let $M$ be a finite length $A$-module and $M^{\vee}=\operatorname{Ext}^{3}(M, A)$. If $M, M^{\prime}$ are two finite length $A$-modules and $f: M \rightarrow M^{\prime}$ an $A$-linear map, then we denote $\operatorname{Ext}^{3}(f, A)$ by $f^{\vee}$. Let

$$
\mathcal{P}=0 \rightarrow P_{3} \stackrel{\partial_{3}}{\rightarrow} P_{2} \stackrel{\partial_{2}}{\rightarrow} P_{1} \stackrel{\partial_{1}}{\rightarrow} P_{0} \stackrel{\theta}{\rightarrow} M \rightarrow 0
$$

be a projective resolution of $M$. Since $\operatorname{Ext}^{i}(M, A)=0$ for $i=0,1,2$ ([5], Th. 18.1), by dualising the above exact sequence we see that

$$
\mathcal{P}^{*}=0 \rightarrow P_{0}^{*} \stackrel{\partial_{1}^{*}}{\rightarrow} P_{1}^{*} \stackrel{\partial_{2}^{*}}{\rightarrow} P_{2}^{*} \stackrel{\partial_{3}^{*}}{\rightarrow} P_{3}^{*} \stackrel{\theta^{\prime}}{\rightarrow} M^{\vee} \rightarrow 0
$$

is a projective resolution of $M^{\vee}$, where $P_{i}^{*}=\operatorname{Hom}_{A}\left(P_{i}, A\right), \partial_{i}^{*}$ is induced by $\partial_{i}$ and for any $f \in P_{3}^{*}$,

$$
\theta^{\prime}(f)=\operatorname{Ext}^{3}(f, M)(\mathcal{P}) \in M^{\vee} .
$$

Throughout this paper, for any surjection $\theta: P_{0} \rightarrow M$ as above, $\theta^{\prime}$ denotes the map defined as above. We define a canonical homomorphism $\mathcal{C}: M \rightarrow M^{\vee \vee}$ as follows : Let $x \in M$. Choose $y \in P_{0}$ such that $\theta(y)=x$. We define 
$\mathcal{C}(x)=\operatorname{Ext}^{3}\left(-e_{y}, M^{\vee}\right)\left(\mathcal{P}^{*}\right) \in M^{\vee \vee}$, where, for $f \in P_{0}^{*}, e_{y}(f)=f(y)$. Then it is easy to see that $\mathcal{C}(x)$ is independent of the choice of $y$ and the diagram

$$
\begin{aligned}
& 0 \quad \rightarrow \quad P_{3} \quad \stackrel{\partial_{3}}{\rightarrow} \quad P_{2} \quad \stackrel{\partial_{2}}{\rightarrow} P_{1} \quad \stackrel{\partial_{1}}{\rightarrow} P_{0} \quad \stackrel{\theta}{\rightarrow} M \quad M \quad \rightarrow \quad 0
\end{aligned}
$$

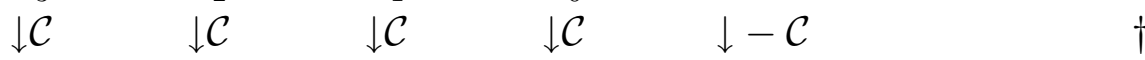

$$
\begin{aligned}
& 0 \rightarrow P_{3}^{* *} \stackrel{\partial_{3}^{* *}}{\longrightarrow} P_{2}^{* *} \stackrel{\partial_{2}^{* *}}{\longrightarrow} P_{1}^{* *} \stackrel{\partial_{1}^{* *}}{\longrightarrow} P_{0}^{* *} \stackrel{\theta^{\prime \prime}}{\longrightarrow} M^{\vee \vee} \rightarrow 0
\end{aligned}
$$

is commutative, where $\mathcal{C}: P_{i} \rightarrow P_{i}^{* *}$ are the canonical isomorphisms. Thus $\mathcal{C}: M \rightarrow M^{\vee \vee}$ is an isomorphism and it is obvious that it is independent of the choice of the projective resolution. We use this isomorphism to identify $M$ with $M^{\vee \vee}$. The choice of the negative sign at $e_{y}$ in the definition of $\mathcal{C}$ is explained in the following: Let $m=\left(x_{1}, x_{2}, x_{3}\right)$ be the maximal ideal of $A$ and

$$
\zeta=0 \rightarrow A \stackrel{\delta_{3}}{\rightarrow} A^{3} \stackrel{\delta_{2}}{\rightarrow} A^{3} \stackrel{\delta_{1}}{\rightarrow} A \stackrel{\eta}{\rightarrow} A / m \rightarrow 0
$$

be the Koszul resolution of $A / m$ with respect to $\left(x_{1}, x_{2}, x_{3}\right)$. With respect to the standard basis $\left\{e_{1}, e_{2}, e_{3}\right\}$ of $A^{3}$, we have

$$
\delta_{1}=\left(\begin{array}{lll}
x_{1} & x_{2} & x_{3}
\end{array}\right), \quad \delta_{2}=\left(\begin{array}{ccc}
-x_{2} & -x_{3} & 0 \\
x_{1} & 0 & -x_{3} \\
0 & x_{1} & x_{2}
\end{array}\right), \quad \delta_{3}=\left(\begin{array}{c}
x_{3} \\
-x_{2} \\
x_{1}
\end{array}\right)
$$

and $\eta: A \rightarrow A / m$ is the natural homomorphism. Let $M$ be a finite dimensional vector space over $A / m$. Then $M$ is a finite length $A$-module. Let $\widetilde{M}=\operatorname{Hom}(M, A / m)$. The assignment $f \mapsto \operatorname{Ext}^{3}(f, A)(\zeta) \in M^{\vee}$ induces a homomorphism

$$
\Phi_{M}: \widetilde{M} \rightarrow M^{\vee} .
$$

The following lemmas are well known, but for the sake of completeness we will give their proofs here.

Lemma 0.1 The homomorphism $\Phi_{M}$ is an isomorphism and the diagram

$$
\begin{array}{lll}
M & \stackrel{\mathcal{C}}{\rightarrow} & M^{\vee \vee} \\
\downarrow \iota & & \downarrow \Phi_{M}^{\vee} \\
\widetilde{\widetilde{M}} & \stackrel{\Phi_{\widetilde{M}}}{\longrightarrow} & (\widetilde{M})^{\vee}
\end{array}
$$

is commutative, where $\iota: M \rightarrow \widetilde{\widetilde{M}}$ is the canonical isomorphism. 
Proof. Since $M \simeq \oplus_{1}^{n} A / m, M^{\vee} \simeq \oplus_{1}^{n}(A / m)^{\vee}$ and $\widetilde{M} \simeq \oplus_{1}^{n} \widetilde{A / m}$, it is enough to prove the lemma in the case when $M=A / m$. In this case it is easy to see that $\Phi_{M} \neq 0$. Since $M^{\vee} \simeq A / m([5]$, Th 18.1) and $\widetilde{M} \simeq A / m$, $\Phi_{M}$ is an isomorphism. We now prove the commutativity of the diagram. For all $x \in M$ and $f \in \widetilde{M}$ we have $\iota(x)(f)=f(x)$ and

$$
\Phi_{\widetilde{M}}(\iota(x))=0 \rightarrow A \stackrel{\delta_{3}}{\rightarrow} A^{3} \stackrel{\delta_{2}}{\rightarrow} A^{3} \stackrel{\delta_{1}}{\rightarrow} A \stackrel{\iota(x)^{-1} \eta}{\rightarrow} A / m \rightarrow 0 .
$$

Let $y \in A$ be such that $\eta(y)=x$. Then we have

$$
\mathcal{C}(x)=0 \rightarrow A \stackrel{-\delta_{1}^{*} e_{y}^{-1}}{\rightarrow} A^{3 *} \stackrel{\delta_{2}^{*}}{\rightarrow} A^{3 *} \stackrel{\delta_{3}^{*}}{\rightarrow} A^{*} \stackrel{\eta^{\prime}}{\rightarrow}(A / m)^{\vee} \rightarrow 0 .
$$

Since $\Phi_{M}$ is an isomorphism, we have

$$
\Phi_{M}^{\vee}(\mathcal{C}(x))=0 \rightarrow A \stackrel{-\delta_{1}^{*} e_{y}^{-1}}{\longrightarrow} A^{3 *} \stackrel{\delta_{2}^{*}}{\longrightarrow} A^{3 *} \stackrel{\delta_{3}^{*}}{\longrightarrow} A^{*} \stackrel{\Phi_{M}^{-1} \eta^{\prime}}{\longrightarrow}(A / m)^{\vee} \rightarrow 0 .
$$

Let $\left\{e_{1}^{*}, e_{2}^{*}, e_{3}^{*}\right\}$ be the dual basis of $A^{3 *}$. For $i=1,2$, let $\theta_{i}: A^{3} \rightarrow A^{3 *}$ be given by the following matrices, with respect to the bases $\left\{e_{1}, e_{2}, e_{3}\right\}$ and $\left\{e_{1}^{*}, e_{2}^{*}, e_{3}^{*}\right\}$.

$$
\theta_{1}=\left(\begin{array}{ccc}
0 & 0 & -y^{-1} \\
0 & y^{-1} & 0 \\
-y^{-1} & 0 & 0
\end{array}\right), \quad \theta_{2}=\left(\begin{array}{ccc}
0 & 0 & y^{-1} \\
0 & -y^{-1} & 0 \\
y^{-1} & 0 & 0
\end{array}\right) .
$$

Let $\theta_{3}: A \rightarrow A^{*}$ be the homomorphism defined by $\theta_{3}(1)=l_{y}$, where $l_{y}(a)=a y$ for all $a \in A$. It is easy to see that the following diagram

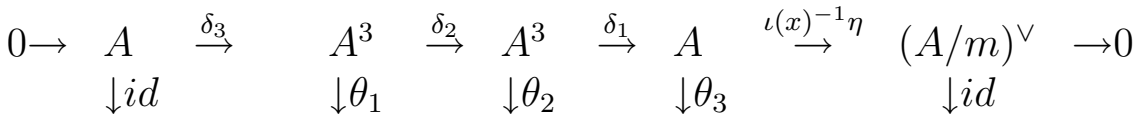

$$
\begin{aligned}
& 0 \rightarrow A \stackrel{-\delta_{1}^{*} e_{y}^{-1}}{\longrightarrow} A^{3 *} \stackrel{\delta_{2}^{*}}{\longrightarrow} A^{3 *} \stackrel{\delta_{3}^{*}}{\longrightarrow} A^{*} \stackrel{\Phi^{-1} \eta^{\prime}}{\longrightarrow}(A / m)^{\vee} \rightarrow 0
\end{aligned}
$$

is commutative. Thus $\Phi_{\widetilde{M}} \iota=\Phi_{M}^{\vee} \mathcal{C}$.

Lemma 0.2 Let $\psi: M \rightarrow \widetilde{M}$ be a homomorphism and $\widetilde{\psi}: \widetilde{\widetilde{M}} \rightarrow \widetilde{M}$ the induced homomorphism. Then the following diagram

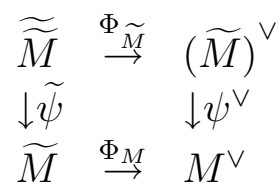

is commutative. 
Proof. Let $f \in \widetilde{\widetilde{M}}$. Then $\Phi_{\widetilde{M}}(f)$ is the pull-back of the Koszul resolution $\zeta$ under $f: \widetilde{M} \rightarrow A / m$ and $\psi^{\vee}\left(\Phi_{\widetilde{M}}(f)\right)$ is the pull-back of the extension $\Phi_{\widetilde{M}}(f)$ under $\psi$. Thus $\psi^{\vee}\left(\Phi_{\widetilde{M}}(f)\right)$ is the pull-back of the Koszul resolution under the homomorphism $f \psi: M \rightarrow A / m$. Since $\widetilde{\psi}(f)=f \psi, \Phi_{M}(\widetilde{\psi})(f)$ is the pull-back of the Koszul resolution under $f \psi$. Thus $\Phi_{M} \psi^{\vee}=\psi^{\vee} \Phi_{\widetilde{M}}$.

The above lemmas enable us to embed the category of $\epsilon$-symmetric spaces on finite dimensional $A / m$-vector spaces into the category of $\epsilon$-symmetric spaces on finite length $A$-modules (cf (0.3)).

Let $\epsilon= \pm 1$. An $\epsilon$-symmetric space of finite length is a pair $(M, \psi)$ where $M$ is a finite length $A$-module and $\psi: M \rightarrow M^{\vee}=\operatorname{Ext}^{3}(M, A)$ is an isomorphism with $\psi^{\vee} \mathcal{C}=\epsilon \psi$. Let $\psi_{1}$ and $\psi_{2}$ be two $\epsilon$-symmetric spaces on finite length $A$-modules $M_{1}$ and $M_{2}$ respectively. We say that $\psi_{1}$ is isometric to $\psi_{2}$, if there exists a homomorphism $\theta: M_{1} \rightarrow M_{2}$ such that $\psi_{1}=\theta^{\vee} \psi_{2} \theta$. An $\epsilon$-symmetric space $\psi$ on $M$ is called metabolic if there exists a submodule $N$ of $M$ such that

$$
0 \rightarrow N \stackrel{i}{\rightarrow} M \stackrel{i^{\vee} \psi}{\rightarrow} N^{\vee} \rightarrow 0
$$

is exact, where $i: N \rightarrow M$ is the inclusion. The Witt group of $\epsilon$-symmetric spaces of finite length $A$-modules is defined as the quotient of the Grothendieck group of isometry classes of $\epsilon$-symmetric spaces with the orthogonal sum as addition, modulo the subgroup generated by metabolic spaces. It is denoted by $W_{\mathrm{fl}}^{\epsilon}(A)$.

Corollary 0.3 Let $M$ be a finite dimensional vector space over $A / m$. Let $\psi: M \rightarrow \widetilde{M}$ be an $\epsilon$-symmetric space, i.e., $\widetilde{\psi} \iota=\psi$ and $\psi$ is an isomorphism. Then $\Phi_{M} \psi: M \rightarrow M^{\vee}$ is an $\epsilon$-symmetric space.

Proof. By (0.1), we have $\left(\Phi_{M} \psi\right)^{\vee} \mathcal{C}=\psi^{\vee} \Phi_{M}^{\vee} \mathcal{C}=\psi^{\vee} \Phi_{\widetilde{M}} \iota$. Using (0.2), we get that $\left(\Phi_{M} \psi\right)^{\vee} \mathcal{C}=\Phi_{M} \widetilde{\psi} \iota=\epsilon \Phi_{M} \psi$. Thus $\Phi_{M} \psi$ is an $\epsilon$-symmetric space.

We need the following

Lemma 0.4 Let $M$ be a finite length $A$-module and $\psi: M \rightarrow M^{\vee}$ an $\epsilon$ symmetric space. If $(M, \psi)$ is stably metabolic, then it is metabolic. 
Proof. If $M$ is an $A / m$-module, then the result follows from the corresponding result for $\epsilon$-symmetric spaces over the field $A / m$. We reduce the general case to the above case by induction on the length of $M$. Assume that the length of $M$ is at least 1 . Let $V$ be a maximal submodule of $M$ which is an $A / m$-module. Suppose that $\psi$ restricted to $V$ is singular. Then there exists a non-zero submodule $L$ of $V$ such that $L \subset L^{\perp}=\operatorname{ker}\left(M \stackrel{i^{\vee} \psi}{\rightarrow} L^{\vee}\right)$ and $\psi$ induces an $\epsilon$-symmetric form $\bar{\psi}$ on $L^{\perp} / L$ which is Witt equivalent to $(M, \psi)$. Suppose that $(M, \psi)$ is stably metabolic. Then $\left(L^{\perp} / L, \bar{\psi}\right)$ is stably metabolic. By induction there exists a submodule $N_{1}$ of $L^{\perp} / L$ such that

$$
0 \rightarrow N_{1} \stackrel{i}{\rightarrow} L^{\perp} / L \stackrel{i^{\vee} \bar{\psi}}{\rightarrow} N_{1}^{\vee} \rightarrow 0
$$

is exact. Let $N$ be the submodule of $M$ containing $L$ such that $N / L=N_{1}$. Then it is easy to see that the sequence

$$
0 \rightarrow N \stackrel{i}{\rightarrow} M \stackrel{i^{\vee} \psi}{\rightarrow} N^{\vee} \rightarrow 0
$$

is exact and $(M, \psi)$ is metabolic. We may therefore assume that $\psi$ restricted to $V$ is non-singular. Then $(M, \psi) \simeq\left(V,\left.\psi\right|_{V}\right) \perp\left(M_{1}, \psi_{1}\right)$. If $M_{1} \neq 0$, then $M_{1}$ contains a non-zero submodule which is an $A / m$-module, contradicting the maximality of $V$. Thus $M_{1}=0$ and $M=V$ is an $A / m$-module. This completes the proof of the lemma.

Let $X$ be a scheme such that 2 is invertible in $\Gamma(X)$. Let $\mathcal{E}$ be a vector bundle over $X$ of finite rank. An $\epsilon$-symmetric space on $\mathcal{E}$ is an isomorphism $\mathbf{q}: \mathcal{E} \rightarrow \mathcal{E}^{*}=\mathcal{H} \operatorname{om}\left(\mathcal{E}, \mathcal{O}_{X}\right)$ such that $\mathbf{q}^{*} \mathcal{C}=\epsilon \mathbf{q}$, where $\mathcal{C}: \mathcal{E} \rightarrow \mathcal{E}^{* *}$ is the canonical identification. Let $W^{\epsilon}(X)$ be the Witt group of $\epsilon$-symmetric spaces on vector bundles over $X([\mathbf{3}], \mathrm{p}$. 144). If $X=\operatorname{Spec}(A)$, then we denote $W^{\epsilon}(X)$ by $W^{\epsilon}(A)$.

Throughout this paper, by an $A$-module we mean a finitely generated $A$-module. We call an $\epsilon$-symmetric space simply a quadratic space if $\epsilon=+1$ and a symplectic space if $\epsilon=-1$. We also denote $W^{+1}(X)$ by $W(X)$. For a vector bundle $\mathcal{E}$ over $X$, we denote the hyperbolic space on $\mathcal{E}$ by $\mathbb{H}(\mathcal{E})([3]$, p. 130). 


\section{Reflexive modules}

Let $A$ be a regular local ring of dimension three with 2 invertible. An $A$ module $E$ is said to be reflexive if it is finitely generated and the canonical homomorphism $E \rightarrow E^{* *}$ is an isomorphism. For a reflexive $A$-module $E$ we use the canonical isomorphism to identify $E^{* *}$ with $E$. It is well-known that a reflexive module over a regular ring of dimension 3 has projective dimension at most 1 . Let $E$ be a reflexive $A$-module and $M=\operatorname{Ext}^{1}\left(E^{*}, A\right)$, where $E^{*}=$ $\operatorname{Hom}_{A}(E, A)$. Since reflexive modules over regular rings of dimension at most 2 are projective, $M$ is a finite length $A$-module. We define a homomorphism $\beta_{E}: \operatorname{Ext}^{1}(E, A) \rightarrow M^{\vee}=\operatorname{Ext}^{3}(M, A)$ as follows. Let

$$
0 \rightarrow P_{1} \stackrel{\partial_{1}}{\rightarrow} P_{0} \stackrel{\partial_{0}}{\rightarrow} E^{*} \rightarrow 0
$$

be a projective resolution of $E^{*}$. Then by dualising, we get an exact sequence

$$
0 \rightarrow E \stackrel{\partial_{0}^{*}}{\rightarrow} P_{0}^{*} \stackrel{\partial_{1}^{*}}{\rightarrow} P_{1}^{*} \stackrel{\delta}{\rightarrow} \operatorname{Ext}^{1}\left(E^{*}, A\right)=M \rightarrow 0,
$$

where $\delta$ is defined by push-outs. We have the following

Lemma 1.1 The Yoneda composition ([4], p. 82) $\beta_{E}: \operatorname{Ext}^{1}(E, A) \rightarrow M^{\vee}$ given by

$$
\beta_{E}\left(0 \rightarrow A \stackrel{\eta}{\rightarrow} Z \stackrel{\eta^{\prime}}{\rightarrow} E \rightarrow 0\right)=\left(0 \rightarrow A \stackrel{\eta}{\rightarrow} Z \stackrel{\partial_{0}^{*} \eta^{\prime}}{\rightarrow} P_{0}^{*} \stackrel{\partial_{1}^{*}}{\rightarrow} P_{1}^{*} \stackrel{\delta}{\rightarrow} M \rightarrow 0\right)
$$

is an isomorphism and is independent of the choice of the projective resolution $(\star)$ of $E^{*}$.

Proof. Consider the long exact sequence of cohomology associated to the short exact sequences

$$
0 \rightarrow E \stackrel{\partial_{0}^{*}}{\rightarrow} P_{0}^{*} \stackrel{\partial_{1}^{*}}{\rightarrow} \operatorname{ker}(\delta) \rightarrow 0 \text { and } 0 \rightarrow \operatorname{ker}(\delta) \hookrightarrow P_{1}^{*} \stackrel{\delta}{\rightarrow} M \rightarrow 0 .
$$

Since $\operatorname{Ext}^{i}(M, A)=0$ for $i \leq 2$ and $P_{1}^{*}$ is a projective module, $\operatorname{Ext}^{1}(\operatorname{ker}(\delta), A)=$ 0 and the connecting homomorphisms $\operatorname{Ext}^{1}(E, A) \rightarrow \operatorname{Ext}^{2}(\operatorname{ker}(\delta), A)$ and $\operatorname{Ext}^{2}(\operatorname{ker}(\delta), A) \rightarrow \operatorname{Ext}^{3}(M, A)$ induced by the above short exact sequences are isomorphisms. Since $\beta_{E}$, up to sign, is the composition of these two connecting homomorphisms ([4], p. 97, Th 9.1), $\beta$ is an isomorphism. 
Suppose that

$$
0 \rightarrow F_{1} \stackrel{\partial_{1}^{\prime}}{\rightarrow} F_{0} \stackrel{\partial_{0}^{\prime}}{\rightarrow} E^{*} \rightarrow 0
$$

is another projective resolution of $E^{*}$. Then by lifting the identity map on $E^{*}$, we get homomorphisms $P_{i} \rightarrow F_{i}, \mathrm{i}=0,1$, such that the diagram

$$
\begin{array}{llllllll}
0 \rightarrow & P_{1} & \stackrel{\partial_{1}}{\rightarrow} & P_{0} & \stackrel{\partial_{0}}{\rightarrow} & E^{*} & \rightarrow 0 \\
& \downarrow & & \downarrow & & \downarrow i d & \\
0 \rightarrow & F_{1} & \stackrel{\partial_{1}^{\prime}}{\rightarrow} & F_{0} & \stackrel{\partial_{0}^{\prime}}{\rightarrow} & E^{*} & \rightarrow 0
\end{array}
$$

is commutative. By dualising this diagram we get a commutative diagram

$$
\begin{array}{lllllllll}
0 \rightarrow & E & \stackrel{\partial_{0}^{\prime *}}{\rightarrow} & F_{0}^{*} & \stackrel{\partial_{1}^{\prime *}}{\rightarrow} & F_{1}^{*} & \stackrel{\delta^{\prime}}{\rightarrow} & M & \rightarrow 0 \\
& \downarrow i d & & \downarrow & & \downarrow & & \downarrow i d & \\
0 \rightarrow & E & \stackrel{\partial_{0}^{*}}{\rightarrow} & P_{0}^{*} & \stackrel{\partial_{1}^{*}}{\rightarrow} & P_{1}^{*} & \stackrel{\delta}{\rightarrow} & M & \rightarrow 0,
\end{array}
$$

where $\delta^{\prime}$ is defined by push-outs. This implies that

$$
\left(0 \rightarrow E \stackrel{\partial_{0}^{\prime *}}{\rightarrow} F_{0}^{*} \stackrel{\partial_{1}^{\prime *}}{\rightarrow} F_{1}^{*} \stackrel{\delta^{\prime}}{\rightarrow} M \rightarrow 0\right)=\left(0 \rightarrow E \stackrel{\partial_{0}^{*}}{\rightarrow} P_{0}^{*} \stackrel{\partial_{1}^{*}}{\rightarrow} P_{1}^{*} \stackrel{\delta}{\rightarrow} M \rightarrow 0\right)
$$

in $\operatorname{Ext}^{2}(M, E)$. Thus the homomorphism $\beta_{E}$ is independent of the choice of the projective resolution of $E^{*}$.

Lemma 1.2 1) For any reflexive $A$-module $E$ we have

$$
\beta_{E^{*}}=-\beta_{E}^{\vee} \mathcal{C}
$$

2) Let $E$ and $E^{\prime}$ be reflexive $A$-modules. Then, for any isomorphism $f$ : $E \rightarrow E^{\prime}$, we have

$$
\operatorname{Ext}^{1}\left(f^{*}\right)^{\vee} \beta_{E^{\prime}}=\beta_{E} \operatorname{Ext}^{1}(f) .
$$

Proof. Let $0 \rightarrow P_{1} \stackrel{\partial_{1}}{\rightarrow} P_{0} \stackrel{\partial_{0}}{\rightarrow} E^{*} \rightarrow 0$ and $0 \rightarrow F_{1} \stackrel{\partial_{1}^{\prime}}{\rightarrow} F_{0} \stackrel{\partial_{0}^{\prime}}{\rightarrow} E \rightarrow 0$ be projective resolutions of $E^{*}$ and $E$ respectively. By dualising these exact sequences, we get exact sequences

$$
0 \rightarrow E \stackrel{\partial_{0}^{*}}{\rightarrow} P_{0}^{*} \stackrel{\partial_{1}^{*}}{\rightarrow} P_{1}^{*} \stackrel{\delta}{\rightarrow} \operatorname{Ext}^{1}\left(E^{*}, A\right) \rightarrow 0
$$


and

$$
0 \rightarrow E^{*} \stackrel{\partial_{0}^{\prime *}}{\rightarrow} F_{0}^{*} \stackrel{\partial_{1}^{\prime *}}{\rightarrow} F_{1}^{*} \stackrel{\delta_{1}}{\rightarrow} \operatorname{Ext}^{1}(E, A) \rightarrow 0 .
$$

Let $\zeta=\left(0 \rightarrow A \stackrel{\alpha}{\rightarrow} Z \stackrel{\beta}{\rightarrow} E^{*} \rightarrow 0\right) \in \operatorname{Ext}^{1}\left(E^{*}, A\right)$. Since $P_{0}$ and $P_{1}$ are projective, there exist homomorphisms $f: P_{1} \rightarrow A$ and $g: P_{0} \rightarrow Z$ such that the diagram

$$
\begin{array}{lllllll}
0 \rightarrow & P_{1} & \stackrel{\partial_{1}}{\rightarrow} & P_{0} & \stackrel{\partial_{0}}{\rightarrow} & E^{*} & \rightarrow 0 \\
& \downarrow f & & \downarrow g & & \downarrow i d & \\
0 \rightarrow & A & \stackrel{\alpha}{\rightarrow} & Z & \stackrel{\beta}{\rightarrow} & E^{*} & \rightarrow 0
\end{array}
$$

is commutative. By the definition of $\delta$ we have $\delta(f)=\zeta$. Since

$$
0 \rightarrow F_{1} \stackrel{\partial_{1}^{\prime}}{\rightarrow} F_{0} \stackrel{\partial_{0}^{*} \partial_{0}^{\prime}}{\rightarrow} P_{0}^{*} \stackrel{\partial_{1}^{*}}{\rightarrow} P_{1}^{*} \stackrel{\delta}{\rightarrow} \operatorname{Ext}^{1}\left(E^{*}, A\right) \rightarrow 0
$$

is a projective resolution of $\operatorname{Ext}^{1}\left(E^{*}, A\right)$, by dualising it we get an exact sequence

$$
0 \rightarrow P_{1} \stackrel{\partial_{1}}{\rightarrow} P_{0} \stackrel{\partial_{0}^{\prime *} \partial_{0}}{\rightarrow} F_{0}^{*} \stackrel{\partial_{1}^{\prime *}}{\rightarrow} F_{1}^{*} \stackrel{\delta_{1}^{\prime}}{\rightarrow} \operatorname{Ext}^{1}\left(E^{*}, A\right)^{\vee} \rightarrow 0 .
$$

Thus $\mathcal{C}(\zeta)=-\xi$, where

$$
\xi=\left(0 \rightarrow A \stackrel{\alpha}{\rightarrow} Z \stackrel{\partial_{0}^{\prime *} \beta}{\rightarrow} F_{0}^{*} \stackrel{\partial_{1}^{\prime *}}{\rightarrow} F_{1}^{*} \stackrel{\delta_{1}^{\prime}}{\rightarrow} \operatorname{Ext}^{1}\left(E^{*}, A\right)^{\vee} \rightarrow 0\right) .
$$

From the definitions of $\delta, \delta^{\prime}$ and $\beta_{E}$, it follows that the diagram

$$
\begin{array}{lllllllcl}
0 \rightarrow & E^{*} & \stackrel{\partial_{0}^{\prime *}}{\rightarrow} & F_{0}^{*} & \stackrel{\partial_{1}^{\prime *}}{\rightarrow} & F_{1}^{*} & \stackrel{\delta_{1}}{\rightarrow} & \operatorname{Ext}^{1}(E, A) & \rightarrow 0 \\
& \downarrow i d & & \downarrow i d & & \downarrow i d & & \downarrow \beta_{E} & \\
0 \rightarrow & E^{*} & \stackrel{\partial_{0}^{\prime *}}{\rightarrow} & F_{0}^{*} & \stackrel{\partial_{1}^{\prime *}}{\rightarrow} & F_{1}^{*} & \stackrel{\delta_{1}^{\prime}}{\rightarrow} & \operatorname{Ext}^{1}\left(E^{*}, A\right)^{\vee} & \rightarrow 0
\end{array}
$$

is commutative. It follows from the definition of $\beta_{E}^{\vee}$ that

$$
\beta_{E}^{\vee}(\xi)=\left(0 \rightarrow A \stackrel{\alpha}{\rightarrow} Z \stackrel{\partial_{0}^{\prime *} \beta}{\rightarrow} F_{0}^{*} \stackrel{\partial_{1}^{\prime *}}{\rightarrow} F_{1}^{*} \stackrel{\delta_{1}}{\rightarrow} \operatorname{Ext}^{1}(E, A) \rightarrow 0\right) .
$$

On the other hand we have

$$
\beta_{E^{*}}(\zeta)=\left(0 \rightarrow A \stackrel{\alpha}{\rightarrow} Z \stackrel{\partial_{0}^{\prime *} \beta}{\rightarrow} F_{0}^{*} \stackrel{\partial_{1}^{\prime *}}{\rightarrow} F_{1}^{*} \stackrel{\delta_{1}}{\rightarrow} \operatorname{Ext}^{1}(E, A) \rightarrow 0\right)=\beta_{E}^{\vee}(\xi) .
$$

Thus $-\beta_{E}^{\vee} \mathcal{C}=\beta_{E^{*}}$. 
Let $f: E \rightarrow E^{\prime}$ be an isomorphism. Then $0 \rightarrow P_{1} \stackrel{\partial_{1}}{\rightarrow} P_{0} \stackrel{f^{*-1} \partial_{0}}{\rightarrow} E^{\prime *} \rightarrow 0$ is a projective resolution of $E^{\prime *}$. By dualising it we get an exact sequence

$$
0 \rightarrow E^{\prime} \stackrel{\partial_{0}^{*} f^{-1}}{\rightarrow} P_{0}^{*} \stackrel{\partial_{1}^{*}}{\rightarrow} P_{1}^{*} \stackrel{\delta_{2}}{\rightarrow} \operatorname{Ext}^{1}\left(E^{* *}, A\right) \rightarrow 0,
$$

with $\delta_{2}=\operatorname{Ext}^{1}\left(f^{*}\right) \delta$. Let $\zeta=\left(0 \rightarrow A \stackrel{\alpha}{\rightarrow} Z \stackrel{\beta}{\rightarrow} E^{\prime} \rightarrow 0\right) \in \operatorname{Ext}^{1}\left(E^{\prime}, A\right)$. Then

$$
\beta_{E^{\prime}}(\zeta)=\left(0 \rightarrow A \stackrel{\alpha}{\rightarrow} Z \stackrel{\partial_{0}^{*} f^{-1} \beta}{\rightarrow} P_{0}^{*} \stackrel{\partial_{1}^{*}}{\rightarrow} P_{1}^{*} \stackrel{\delta_{2}}{\rightarrow} \operatorname{Ext}^{1}\left(E^{\prime *}, A\right) \rightarrow 0\right)
$$

and

$$
\operatorname{Ext}^{1}\left(f^{*}\right)^{\vee} \beta_{E^{\prime}}(\zeta)=\left(0 \rightarrow A \stackrel{\alpha}{\rightarrow} Z \stackrel{\partial_{0}^{*} \stackrel{f^{-1} \beta}{\rightarrow}}{\rightarrow} P_{0}^{*} \stackrel{\partial_{1}^{*}}{\rightarrow} P_{1}^{*} \stackrel{\delta}{\rightarrow} \operatorname{Ext}^{1}\left(E^{*}, A\right) \rightarrow 0\right),
$$

since $\delta=\operatorname{Ext}^{1}\left(f^{*}\right)^{-1} \delta_{2}$. On the other hand we have

$$
\operatorname{Ext}^{1}(f)(\zeta)=\left(0 \rightarrow A \stackrel{\alpha}{\rightarrow} Z \stackrel{f^{-1} \beta}{\rightarrow} E \rightarrow 0\right)
$$

and

$$
\begin{aligned}
\beta_{E} \operatorname{Ext}^{1}(f)(\zeta) & =\left(0 \rightarrow A \stackrel{\alpha}{\rightarrow} Z \stackrel{\partial_{0}^{*} \stackrel{f^{-1} \beta}{\rightarrow}}{\rightarrow} P_{0}^{*} \stackrel{\partial_{1}^{*}}{\rightarrow} P_{1}^{*} \stackrel{\delta}{\rightarrow} \operatorname{Ext}^{1}\left(E^{*}, A\right) \rightarrow 0\right) \\
& =\operatorname{Ext}^{1}\left(f^{*}\right)^{\vee} \beta_{E^{\prime}}(\zeta) .
\end{aligned}
$$

This proves the lemma.

Let $A$ be any local ring in which 2 is invertible and $m$ its maximal ideal. Let $E$ be a reflexive $A$-module. By an $\epsilon$-symmetric space on $E$ we mean an isomorphism $q: E \rightarrow E^{*}$ such that $q^{*} \mathcal{C}=\epsilon q$, where $\mathcal{C}: E \rightarrow E^{* *}$ is the canonical isomorphism.

Let $V$ be an $A$-module. By a unimodular element of $V$ we mean an element $x \in V$ such that $f(x)=1$ for some $A$-linear map $f: V \rightarrow A$. For example, an element $\left(a_{1}, \cdots, a_{n}\right) \in A^{n}$ is unimodular if and only if $a_{i} \notin m$ for some $i$. Thus, if an $A$-module $V$ has no unimodular elements and $\eta: V \rightarrow A^{n}$ is an $A$-linear map, then $\eta(V) \subset m A^{n}$.

Lemma 1.3 Let $E$ be a reflexive $A$-module and $q$ an $\epsilon$-symmetric space on $E$. Suppose that $E=E_{0} \oplus A^{n}$ with $E_{0}$ having no unimodular elements. Then there exist $\epsilon$-symmetric spaces $q_{1}$ and $q_{2}$ over $E_{0}$ and $A^{n}$ respectively such that

$$
(E, q) \simeq\left(E_{0}, q_{1}\right) \perp\left(A^{n}, q_{2}\right)
$$


Proof. Let $E=E_{0} \oplus A^{n}$ be such that $E_{0}$ has no unimodular elements. Then

$$
q=\left(\begin{array}{cc}
q_{1} & \eta \\
\epsilon \eta^{*} & q_{1}^{\prime}
\end{array}\right)
$$

for some $q_{1}: E_{0} \rightarrow E_{0}^{*}, q_{1}^{\prime}: A^{n} \rightarrow A^{n *}$ and $\eta: A^{n} \rightarrow E_{0}^{*}$. Since $E_{0}$ has no unimodular elements, $\eta^{*}\left(E_{0}\right) \subset m A^{n *}$ and hence $\eta\left(A^{n}\right) \subset m E_{0}^{*}$. This implies that

$$
q \equiv\left(\begin{array}{cc}
q_{1} & 0 \\
0 & q_{1}^{\prime}
\end{array}\right) \bmod m E^{*}
$$

Since $q$ is an isomorphism, $q_{1}$ and $q_{1}^{\prime}$ are isomorphisms. We have

$$
\left(\begin{array}{cc}
1 & 0 \\
-\epsilon \eta^{*} q_{1}^{-1} & 1
\end{array}\right)\left(\begin{array}{cc}
q_{1} & \eta \\
\epsilon \eta^{*} & q_{1}^{\prime}
\end{array}\right)\left(\begin{array}{cc}
1 & -q_{1}^{-1} \eta \\
0 & 1
\end{array}\right)=\left(\begin{array}{cc}
q_{1} & 0 \\
0 & -\eta^{*} q_{1}^{-1} \eta+q_{1}^{\prime}
\end{array}\right)
$$

Let $q_{2}=-\eta^{*} q_{1}^{-1} \eta+q_{1}^{\prime}: A^{n} \rightarrow A^{n *}$. Since $q_{1}^{*}=\epsilon q_{1},(E, q) \simeq\left(E_{0}, q_{1}\right) \perp\left(A^{n}, q_{2}\right)$.

\section{Spaces over the punctured spectrum and on finite length modules}

We begin by recalling from a paper of Horrocks $([\mathbf{2}])$ an equivalence between the categories $\Phi \mathcal{P}$ of $\Phi$-equivalence classes of vector bundles on the punctured spectrum of a regular local ring $A$ of dimension 3 and the category $\mathcal{M}$ of finite length $A$-modules. Let $m$ be the maximal ideal of $A$ and $Y=\operatorname{Spec}(A) \backslash\{m\}$. Let $\mathcal{E}$ be a vector bundle over $Y$ and $E=\Gamma(\mathcal{E})$ the module of sections of $\mathcal{E}$. Then $E$ is a reflexive $A$-module $\left([2]\right.$, Th.4.1) and $M=\operatorname{Ext}^{1}\left(E^{*}, A\right)$, which is isomorphic to $H^{1}(Y, \mathcal{E})([\mathbf{2}], \S 5)$, is a finite length $A$-module ([2], Cor.7.2.5). The functor $T: \Phi \mathcal{P} \rightarrow \mathcal{M}$ given by $T(\mathcal{E})=\operatorname{Ext}^{1}\left(E^{*}, A\right)$ is an equivalence of categories ([2], Cor. 7.2.5). Let $M$ be a finite length $A$-module. The construction below gives a vector bundle $\mathcal{E}$ on $Y$ such that $T(\mathcal{E})=M$. Let, in fact

$$
0 \rightarrow P_{3} \stackrel{\partial_{3}}{\rightarrow} P_{2} \stackrel{\partial_{2}}{\rightarrow} P_{1} \stackrel{\partial_{1}}{\rightarrow} P_{0} \stackrel{\eta}{\rightarrow} M \rightarrow 0
$$

be a projective resolution of $M$. Let $E=\operatorname{ker}\left(\partial_{1}\right)$. Then $E$ is an $A$-module of projective dimension at most 1 and $\operatorname{Ext}^{1}\left(E^{*}, A\right)=M$. Since $M$ is a finite length module, for any prime ideal $p$ of $A, p \neq m, M_{p}=0$ and hence $E_{p}$ is free. Thus $E=\Gamma(\mathcal{E})$ for some vector bundle $\mathcal{E}$ on $Y([\mathbf{2}]$, Th. 4.1). 
Let $A$ be a regular local ring of dimension 3 in which 2 is invertible. Let $\mathcal{E}$ be a vector bundle and $\mathbf{q}$ an $\epsilon$-symmetric space on $\mathcal{E}$. We associate to $(\mathcal{E}, \mathbf{q})$ a $(-\epsilon)$-symmetric space $\rho(\mathbf{q})$ of finite length. The $\epsilon$-symmetric space q on $\mathcal{E}$ gives rise to an $\epsilon$-symmetric space $(E, q)$, where $E=\Gamma(\mathcal{E})$. Then $M=\operatorname{Ext}^{1}\left(E^{*}, A\right)$ is a finite length $A$-module. The isomorphism $q: E \rightarrow E^{*}$ induces an isomorphism $\operatorname{Ext}^{1}(q): M=\operatorname{Ext}^{1}\left(E^{*}, A\right) \rightarrow \operatorname{Ext}^{1}(E, A)$. Let $\rho(\mathbf{q})=$ $\beta_{E} \operatorname{Ext}^{1}(q)$. We have the following

Lemma 2.1 $\rho(\mathbf{q}): M \rightarrow M^{\vee}$ is a $(-\epsilon)$-symmetric space.

Proof. In the following diagram

$$
\begin{array}{ccccc}
M=\operatorname{Ext}^{1}\left(E^{*}, A\right) & \stackrel{\operatorname{Ext}^{1}(q)}{\rightarrow} & \operatorname{Ext}^{1}(E, A) & \stackrel{\beta_{E}}{\rightarrow} & \operatorname{Ext}^{1}\left(E^{*}, A\right)^{\vee}=M^{\vee} \\
\downarrow \beta_{E^{*}} & & \downarrow \beta_{E} & & \downarrow \text { id } \\
\operatorname{Ext}^{1}(E, A)^{\vee} & \epsilon \operatorname{Ext}^{1}(q)^{\vee} & \operatorname{Ext}^{1}\left(E^{*}, A\right)^{\vee} & \stackrel{i d}{\rightarrow} & \operatorname{Ext}^{1}\left(E^{*}, A\right)^{\vee} \\
\downarrow \beta_{E}^{\vee-1} & & \downarrow \epsilon \operatorname{Ext}^{1}(q)^{\vee-1} & & \downarrow \text { id } \\
\operatorname{Ext}^{1}\left(E^{*}, A\right)^{\vee \vee} & \stackrel{\beta_{E}^{\vee}}{\rightarrow} & \operatorname{Ext}^{1}(E, A)^{\vee} & \underset{\operatorname{Ext}^{1}(q)^{\vee}}{\rightarrow} & \operatorname{Ext}^{1}\left(E^{*}, A\right)^{\vee}
\end{array}
$$

clearly all the squares except perhaps the top left one commutes. Since $q^{*}=$ $\epsilon q$, by (1.2) this square also commutes. By (1.2), the composition of maps on the first column is equal to $-\mathcal{C}$. Thus

$$
\rho(q)^{\vee} \mathcal{C}=\operatorname{Ext}^{1}(q)^{\vee} \beta_{E}^{\vee} \mathcal{C}=-\epsilon \beta_{E} \operatorname{Ext}^{1}(q)=-\epsilon \rho(q) .
$$

Lemma 2.2 Let $M$ be a finite length $A$-module and $\psi$ an $\epsilon$-symmetric form on $M$. Suppose that there exists an exact sequence

$$
N \stackrel{f}{\rightarrow} M \stackrel{f^{\vee} \psi}{\rightarrow} N^{\vee}
$$

of finite length $A$-modules. Then $(M, \psi)$ is metabolic.

Proof. Since the map $f$ factors as $N \rightarrow N / \operatorname{ker}(\mathrm{f}) \stackrel{\bar{f}}{\rightarrow} M$, we have an exact sequence

$$
0 \rightarrow N / \operatorname{ker}(\mathrm{f}) \stackrel{\bar{f}}{\rightarrow} M \stackrel{\bar{f}^{\vee} \psi}{\rightarrow}(N / \operatorname{ker}(\mathrm{f}))^{\vee} .
$$

Since, dimension of $A$ being $3, \operatorname{Ext}^{4}(L, A)=0$, the map $\bar{f}^{\vee} \psi$ is surjective and hence $(M, \psi)$ is metabolic. 
Lemma 2.3 If $(\mathcal{E}, \mathbf{q})$ is metabolic, then $(M, \rho(\mathbf{q}))$ is metabolic.

Proof. Suppose that $(\mathcal{E}, \mathbf{q})$ is metabolic. Let $\mathcal{F}$ be a subbundle of $\mathcal{E}$ such that the sequence

$$
0 \rightarrow \mathcal{F} \stackrel{i}{\rightarrow} \mathcal{E} \stackrel{i^{*} \mathbf{q}}{\rightarrow} \mathcal{F}^{*} \rightarrow 0
$$

is exact, where $\mathcal{F} \stackrel{i}{\rightarrow} \mathcal{E}$ is the inclusion. By taking global sections and then applying the Ext functor to the following exact sequence of bundles

$$
0 \rightarrow \mathcal{F} \stackrel{\mathbf{q}^{i}}{\rightarrow} \mathcal{E}^{*} \stackrel{i^{*}}{\rightarrow} \mathcal{F}^{*} \rightarrow 0
$$

we get an exact sequence

$$
\operatorname{Ext}^{1}\left(F^{*}, A\right) \rightarrow \operatorname{Ext}^{1}\left(E^{*}, A\right) \rightarrow \operatorname{Ext}^{1}(F, A)
$$

of finite length modules, where $F=\Gamma(\mathcal{F})$. Let $N=\operatorname{Ext}^{1}\left(F^{*}, A\right)$. Then the canonical identification of $\operatorname{Ext}^{1}(F, A)$ with $N^{\vee}$ gives an exact sequence

$$
N \stackrel{f}{\rightarrow} M \stackrel{f^{\vee} \stackrel{\rho(\mathbf{q})}{\rightarrow}}{\rightarrow} N^{\vee}
$$

Now the lemma follows from (1.2).

Lemma 2.4 The assignment $(\mathcal{E}, \mathbf{q}) \mapsto(M, \rho(\mathbf{q}))$ induces a homomorphism

$$
\rho: W^{\epsilon}(Y) \rightarrow W_{f l}^{-\epsilon}(A)
$$

Proof. Since $\rho$ is clearly additive, it is enough to show that $\rho$ takes stably metabolic spaces to metabolic spaces. Let $(\mathcal{E}, \mathbf{q})$ be an $\epsilon$-symmetric spaces over $Y$ which is stably metabolic. Then there exists a metabolic space $\left(\mathcal{E}_{1}, \mathbf{q}_{1}\right)$ such that $(\mathcal{E}, \mathbf{q}) \perp\left(\mathcal{E}_{1}, \mathbf{q}_{1}\right)$ is metabolic. By $(2.3), \rho\left(\mathbf{q}_{1}\right)$ and $\rho\left(\mathbf{q} \perp \mathbf{q}_{1}\right)=$ $\rho(\mathbf{q}) \perp \rho\left(\mathbf{q}_{1}\right)$ are metabolic. Thus $\rho(\mathbf{q})$ is stably metabolic.

We note that if $\mathcal{E}$ is a trivial bundle then $M=0$. Thus, if $(\mathcal{E}, \mathbf{q})$ comes from an $\epsilon$-symmetric space on $A$, then $\rho(\mathbf{q})=0$.

The proof of the following lemma is by straightforward verification, hence we omit it. 
Lemma 2.5 Let $R$ be a ring. Let $0 \rightarrow N \stackrel{i}{\rightarrow} M \stackrel{j}{\rightarrow} N^{\prime} \rightarrow 0$ be an exact sequence of $R$-modules. Assume that the projective dimensions of $N$ and $N^{\prime}$ are finite. Let

$$
0 \rightarrow P_{n} \stackrel{\partial_{n}}{\rightarrow} P_{n-1} \stackrel{\partial_{n-1}}{\rightarrow} \cdots P_{1} \stackrel{\partial_{1}}{\rightarrow} P_{0} \stackrel{\alpha}{\rightarrow} N \rightarrow 0
$$

and

$$
0 \rightarrow Q_{n} \stackrel{\partial_{n}^{\prime}}{\rightarrow} Q_{n-1} \stackrel{\partial_{n-1}^{\prime}}{\rightarrow} \cdots \rightarrow Q_{1} \stackrel{\partial_{1}^{\prime}}{\rightarrow} Q_{0} \stackrel{\beta}{\rightarrow} N^{\prime} \rightarrow 0
$$

be projective resolutions of $N$ and $N^{\prime}$ respectively. Let, for $l \geq 1, \phi_{l}$ : $Q_{l} \rightarrow P_{l-1}$ and $\theta: Q_{0} \rightarrow M$ be $R$-linear homomorphisms. Let

$$
\delta_{l}=\left(\begin{array}{cc}
\partial_{l} & (-1)^{l} \phi_{l} \\
0 & \partial_{l}^{\prime}
\end{array}\right)
$$

Then the following diagram

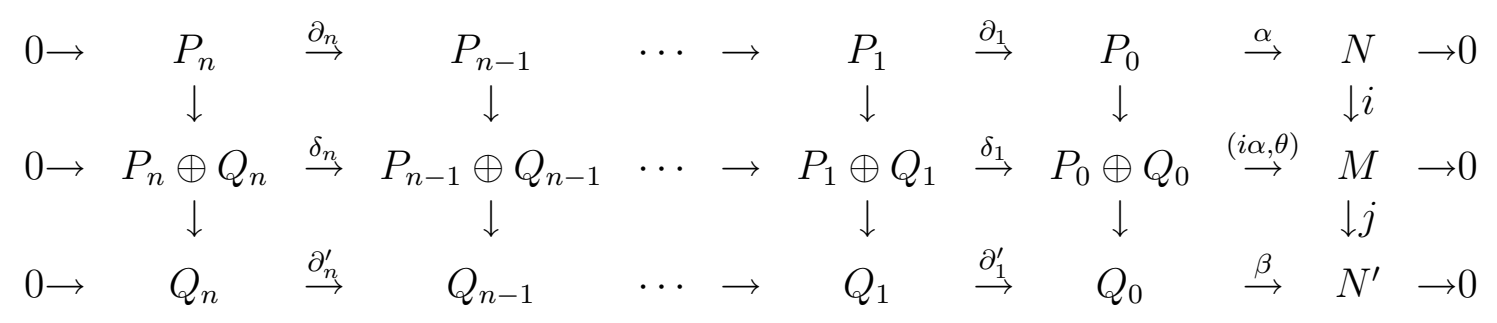

is commutative if and only if the following diagram

$$
\begin{aligned}
& 0 \quad \rightarrow \quad Q_{n} \quad \stackrel{\partial_{n}^{\prime}}{\rightarrow} Q_{n-1} \stackrel{\partial_{n-1}^{\prime}}{\rightarrow} \cdots \rightarrow Q_{2} \stackrel{\partial_{2}^{\prime}}{\rightarrow} Q_{1} \stackrel{\stackrel{\partial_{1}^{\prime}}{\rightarrow}}{\rightarrow} Q_{0} \stackrel{\beta}{\rightarrow} N^{\prime} \quad \rightarrow 0 \\
& \downarrow \quad \downarrow \phi_{n} \quad \downarrow \phi_{n-1} \quad \downarrow \phi_{2} \quad \downarrow \phi_{1} \quad \downarrow \theta \quad P_{n} \quad \downarrow i d \\
& 0 \rightarrow P_{n} \stackrel{\partial_{n}}{\rightarrow} P_{n-1} \stackrel{\partial_{n-1}}{\rightarrow} P_{n-2} \quad \stackrel{\partial_{n-2}}{\rightarrow} \quad \cdots \quad \rightarrow \quad P_{1} \quad \stackrel{\partial_{1}}{\rightarrow} P_{0} \quad \stackrel{i \alpha}{\rightarrow} M \quad M \quad \stackrel{j}{\rightarrow} N^{\prime} \rightarrow 0
\end{aligned}
$$

is commutative.

Proposition 2.6 Let $(\mathcal{E}, \mathbf{q})$ be an $\epsilon$-symmetric space over $Y$. Suppose that $E=\Gamma(\mathcal{E})$ has no unimodular elements and that $\rho(\mathbf{q})$ is metabolic. Then there exist $\epsilon$-symmetric spaces $\mathbf{q}_{1}$ and $\mathbf{q}_{2}$ on $\mathcal{E}$ and $\mathcal{O}_{Y}{ }^{n}$ respectively such that $\mathbf{q}_{1} \perp \mathbf{q}_{2}$ is metabolic and $\rho(\mathbf{q}) \simeq \rho\left(\mathbf{q}_{1}\right)$. 
Proof. Let $M=\operatorname{Ext}^{1}\left(E^{*}, A\right)$ and $\rho(\mathbf{q})$ be the $\epsilon$-symmetric space on $M$. Since $\rho(\mathbf{q})$ is metabolic, there exists an exact sequence

$$
0 \rightarrow N \stackrel{i}{\rightarrow} M \stackrel{i^{\vee} \rho(q)}{\rightarrow} N^{\vee} \rightarrow 0
$$

Let

$$
0 \rightarrow Q_{3} \stackrel{\partial_{3}}{\rightarrow} Q_{2} \stackrel{\partial_{2}}{\rightarrow} Q_{1} \stackrel{\partial_{1}}{\rightarrow} Q_{0} \stackrel{\eta}{\rightarrow} N \rightarrow 0
$$

be a projective resolution of $N$. By dualising this resolution, we get a projective resolution

$$
0 \rightarrow Q_{0}^{*} \stackrel{\partial_{1}^{*}}{\rightarrow} Q_{1}^{*} \stackrel{\partial_{2}^{*}}{\rightarrow} Q_{2}^{*} \stackrel{\partial_{3}^{*}}{\rightarrow} Q_{3}^{*} \stackrel{\eta^{\prime}}{\rightarrow} N^{\vee} \rightarrow 0
$$

of $N^{\vee}$. By lifting the identity map of $N^{\vee}$, we obtain a commutative diagram

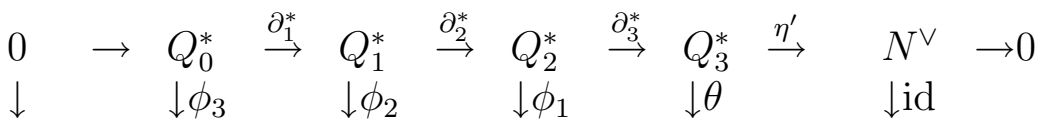

$$
\begin{aligned}
& Q_{3} \quad \stackrel{\partial_{3}}{\rightarrow} Q_{2} \quad \stackrel{\partial_{2}}{\rightarrow} Q_{1} \quad \stackrel{\partial_{1}}{\rightarrow} Q_{0} \stackrel{i \eta}{\rightarrow} M \stackrel{i^{\vee} \rho(q)}{\rightarrow} N^{\vee} \rightarrow 0 .
\end{aligned}
$$

Let

$$
\delta_{1}=\left(\begin{array}{cc}
\partial_{1} & -\phi_{1} \\
0 & \partial_{3}^{*}
\end{array}\right), \quad \delta_{2}=\left(\begin{array}{cc}
\partial_{2} & \phi_{2} \\
0 & \partial_{2}^{*}
\end{array}\right), \quad \delta_{3}=\left(\begin{array}{cc}
\partial_{3} & -\phi_{3} \\
0 & \partial_{1}^{*}
\end{array}\right) .
$$

By (2.5), the diagram

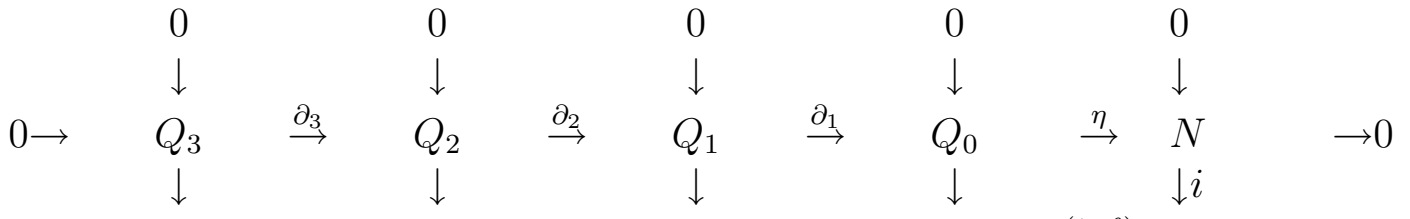

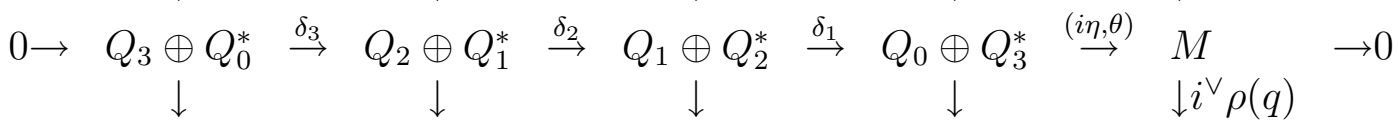

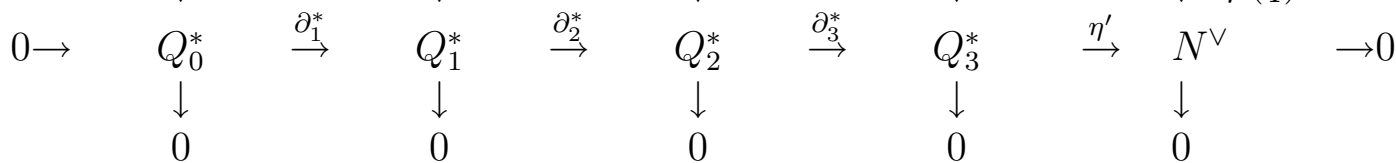

is commutative. Since the first row, the last rows and all the columns in the above diagram are exact and the diagram is commutative, from the long 
exact homology sequence $([4]$, Th. 4.1 p. 45$)$ we get that the middle row is also exact. By dualising this diagram, we get the commutative diagram

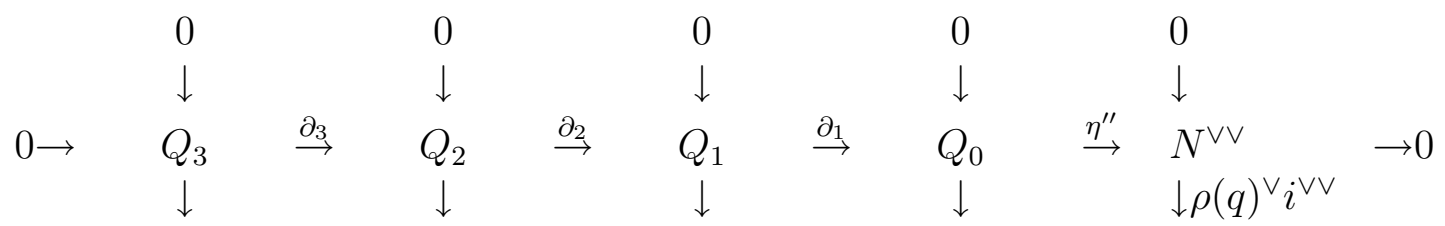

$$
\begin{aligned}
& 0 \rightarrow Q_{3} \oplus Q_{0}^{*} \stackrel{\delta_{1}^{*}}{\rightarrow} Q_{2} \oplus Q_{1}^{*} \stackrel{\delta_{2}^{*}}{\rightarrow} Q_{1} \oplus Q_{2}^{*} \stackrel{\delta_{3}^{*}}{\rightarrow} Q_{0} \oplus Q_{3}^{*} \stackrel{\stackrel{(\mu, \nu)}{\rightarrow}}{\rightarrow} M^{\vee} \quad \rightarrow 0
\end{aligned}
$$

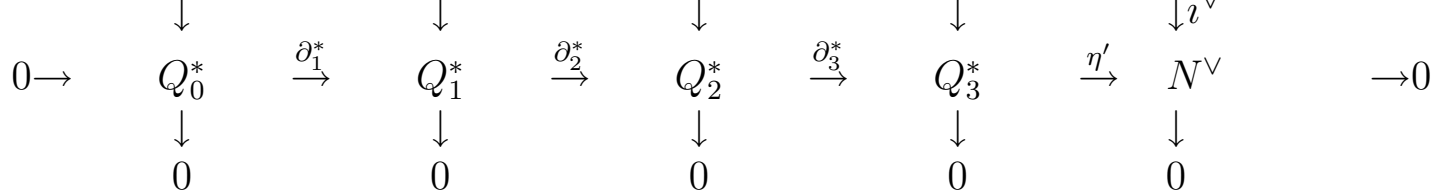

with exact rows and columns. By (2.5), the diagram

$$
\begin{array}{llllllllllllll}
0 & \rightarrow & Q_{0}^{*} & \stackrel{\partial_{1}^{*}}{\rightarrow} & Q_{1}^{*} & \stackrel{\partial_{2}^{*}}{\rightarrow} & Q_{2}^{*} & \stackrel{\partial_{3}^{*}}{\rightarrow} & Q_{3}^{*} & \stackrel{\eta^{\prime}}{\rightarrow} & N^{\vee} & \rightarrow 0 \\
\downarrow & & \downarrow \phi_{1}^{*} & & \downarrow \phi_{2}^{*} & & \downarrow \phi_{3}^{*} & & & & & & & \\
Q_{3} & \stackrel{\partial_{3}}{\rightarrow} & Q_{2} & \stackrel{\partial_{2}}{\rightarrow} & Q_{1} & \stackrel{\partial_{1}}{\rightarrow} & Q_{0} & \stackrel{\rho(q)^{\vee} i^{\vee}}{\rightarrow} \eta^{\prime \prime} & M^{\vee} & \stackrel{i^{\vee}}{\rightarrow} & N^{\vee} & \rightarrow 0
\end{array}
$$

is commutative. From the definition of $\eta^{\prime \prime}$ and $\mathcal{C}: N \rightarrow N^{\vee \vee}$ (cf diagram $\dagger$ in $\S 0)$ it follows that $\mathcal{C} \eta=-\eta^{\prime \prime}$. Since $\rho(q)^{\vee} \mathcal{C}=-\epsilon \rho(q)$, we have the commutative diagram

$$
\begin{array}{llllllllllll}
0 & \rightarrow & Q_{0}^{*} & \stackrel{\partial_{1}^{*}}{\rightarrow} & Q_{1}^{*} & \stackrel{\partial_{2}^{*}}{\rightarrow} & Q_{2}^{*} & \stackrel{\partial_{3}^{*}}{\rightarrow} & Q_{3}^{*} & \stackrel{\eta^{\prime}}{\rightarrow} & N^{\vee} & \rightarrow 0 \\
\downarrow & & \downarrow \phi_{1}^{*} & & \downarrow \phi_{2}^{*} & & \downarrow \phi_{3}^{*} & & \downarrow \nu^{\prime} & & \downarrow \in \mathrm{id} & \\
Q_{3} & \stackrel{\partial_{3}}{\rightarrow} & Q_{2} & \stackrel{\partial_{2}}{\rightarrow} & Q_{1} & \stackrel{\partial_{1}}{\rightarrow} & Q_{0} & \stackrel{i \eta}{\rightarrow} & M & \stackrel{i^{\vee} \rho(q)}{\rightarrow} & N^{\vee} & \rightarrow 0
\end{array}
$$

where $\nu^{\prime}=\epsilon \rho(q)^{-1} \nu$. From $(\star)$ and the above commutative diagram we get maps $s_{1}: Q_{2}^{*} \rightarrow Q_{1}$ and $s_{2}: Q_{1}^{*} \rightarrow Q_{2}$ such that $\phi_{2}-\epsilon \phi_{2}^{*}=\partial_{2} s_{2}-s_{1} \partial_{2}^{*}$. Let $\phi=\phi_{2}-\epsilon \partial_{2} s_{1}^{*}$. Then we have

$$
\begin{aligned}
\partial_{1} \phi & =\partial_{1} \phi_{2} \\
& =\partial_{1}\left(\phi_{2}-\epsilon \phi_{2}^{*}\right) \\
& =\partial_{1}\left(\partial_{2} s_{2}-s_{1} \partial_{2}^{*}\right)+\epsilon \partial_{1} \phi_{2}^{*} \\
& =\epsilon \partial_{1}\left(\phi_{2}^{*}-\epsilon_{1} \partial_{2}^{*}\right) \\
& =\epsilon \partial_{1} \phi^{*} .
\end{aligned}
$$


Let

$$
\delta=\left(\begin{array}{cc}
\partial_{2} & \frac{\phi+\epsilon \phi^{*}}{2} \\
0 & \epsilon \partial_{2}^{*}
\end{array}\right)
$$

It is easy to see that the diagram

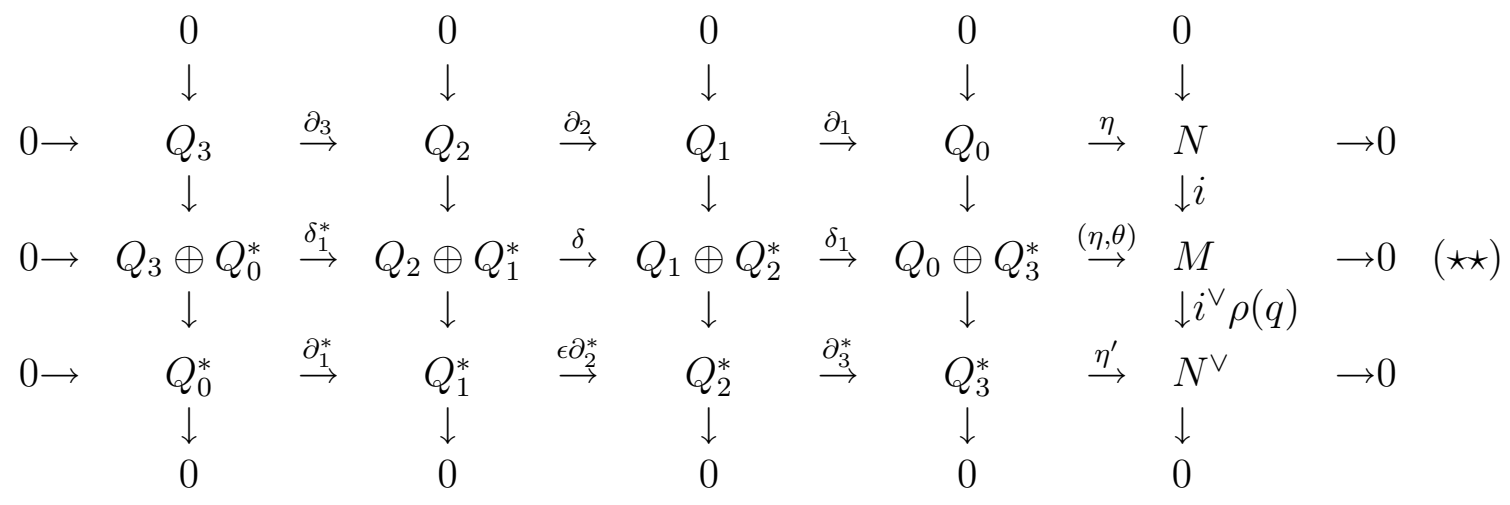

commutes. Since the first row, the last row and all the columns are exact, the middle row is also exact. Let $E^{\prime}=\operatorname{ker}\left(\delta_{1}\right)$. Since $\delta^{*} \mathcal{C}=\epsilon \delta$, from the middle row of the diagram $(\star \star)$ it is easy to see that $\delta$ induces an $\epsilon$-symmetric isomorphism $q^{\prime}: E^{\prime} \rightarrow E^{\prime *}$. Let $\left(\mathcal{E}^{\prime}, \mathbf{q}^{\prime}\right)$ be the $\epsilon$-symmetric space over $Y$ with $\Gamma\left(\mathcal{E}^{\prime}\right)=E^{\prime}$ and $\Gamma\left(\mathbf{q}^{\prime}\right)=q^{\prime}$. Since $\operatorname{Ext}^{1}\left(E^{\prime}, A\right) \simeq M=\operatorname{Ext}^{1}\left(E^{*}, A\right)$ and $E$ has no unimodular elements, by Cor. 7.2.5 and Lemma 7.1 of [2] we have $E^{\prime}=$ $E \oplus A^{n}$. Then by $(1.3),\left(E^{\prime}, q^{\prime}\right) \simeq\left(E, q_{1}\right) \perp\left(A^{n}, q_{2}\right)$ for some $\epsilon$-symmetric spaces $q_{1}$ and $q_{2}$ on $E$ and $A^{n}$ respectively. Let $\mathbf{q}_{1}$ be the $\epsilon$-symmetric space on $\mathcal{E}$ such that $\Gamma\left(\mathbf{q}_{1}\right)=q_{1}$. Let $F=\operatorname{ker}\left(\partial_{1}\right)$ and $\mathcal{F}$ the vector bundle over $Y$ with $\Gamma(\mathcal{F})=F$. Then using $(\star \star)$, it is easy to see that $\mathcal{F}$ is a Lagrangian for $\left(\mathcal{E}^{\prime}, \mathbf{q}^{\prime}\right) \simeq\left(\mathcal{E}, \mathbf{q}_{1}\right) \perp\left(\mathcal{O}_{Y}{ }^{n}, \mathbf{q}_{2}\right)$, where $\Gamma\left(\mathbf{q}_{2}\right)=q_{2}$. Since the map $E^{\prime} \rightarrow F^{*}$ induced by the diagram $(\star \star)$, induces $i^{\vee} \rho(\mathbf{q}): M \rightarrow N^{\vee}$, we have $i^{\vee} \rho(\mathbf{q})=$ $i^{\vee} \rho\left(\mathbf{q}_{1}\right)$. Thus, by the lemma (2.7) below, we have $\rho(\mathbf{q}) \simeq \rho\left(\mathbf{q}_{1}\right)$.

Lemma 2.7 Let $\psi_{1}$ and $\psi_{2}$ be two $\epsilon$-symmetric spaces on $M$. Suppose there exists a submodule $N$ such that

$$
0 \rightarrow N \stackrel{i}{\rightarrow} M \stackrel{i^{\vee} \psi_{1}}{\rightarrow} N^{\vee} \rightarrow 0
$$

is exact and $i^{\vee} \psi_{1}=i^{\vee} \psi_{2}$. Then $\psi_{1} \simeq \psi_{2}$. 
Proof. Since $i^{\vee} \psi_{1}=i^{\vee} \psi_{2}$, there exists a map $\theta: M \rightarrow N$ such that $\psi_{1}^{-1} \psi_{2}-$ $1=i \theta$, i.e., $\psi_{1} i \theta=\psi_{2}-\psi_{1}=\theta^{\vee} i^{\vee} \psi_{1}$. We have

$$
\begin{aligned}
\frac{\left(1+\theta^{\vee} i^{\vee}\right)}{2} \psi_{1} \frac{(1+i \theta)}{2} & =\frac{\left(\psi_{1}+\theta^{\vee} i^{\vee} \psi_{1}\right)}{2} \frac{(1+i \theta)}{2} \\
& =\psi_{1}+\psi_{1} \frac{i \theta}{2}+\frac{\theta^{\vee} i^{\vee}}{2} \psi_{1}+\frac{\theta^{\vee} i^{\vee}}{2} \psi_{1} \frac{i \theta}{2} \\
& =\psi_{1}+\frac{\psi_{2}-\psi_{1}}{2}+\frac{\psi_{2}-\psi_{1}}{2} \\
& =\psi_{2}
\end{aligned}
$$

\section{The Witt groups of the punctured spec- trum and purity}

Let $A$ be a regular local ring of dimension three with 2 invertible. Let $Y=$ $\operatorname{Spec}(A) \backslash\{m\}$, where $m$ is the maximal ideal of $A$.

Proposition 3.1. Let $\mathcal{E}$ be a vector bundle on $Y$ and $\mathbf{q}: \mathcal{E} \rightarrow \mathcal{E}^{*}$ an $\epsilon$ symmetric isomorphism. Suppose that $\Gamma(\mathcal{E})$ has no unimodular elements. If $\rho(\mathbf{q})$ is isomorphic to a hyperbolic space, then $\mathbf{q}$ is in the image of $W^{\epsilon}(A)$.

Proof. Let $N$ be a finite length $A$-module such that $(M, \rho(\mathbf{q}))$ is isomorphic to the hyperbolic space $\mathbb{I}(N)$. Let $\mathcal{F}$ be the vector bundle on $Y$ with $\Gamma(\mathcal{F})$ having no unimodular elements and such that $H^{1}(Y, \mathcal{F}) \simeq N$ (cf $\left.\S 2\right)$. Since $H^{1}(Y, \mathcal{E}) \simeq N \oplus N^{\vee} \simeq H^{1}\left(Y, \mathcal{F} \oplus \mathcal{F}^{*}\right)$ with $\Gamma(\mathcal{E})$ and $\Gamma\left(\mathcal{F} \oplus \mathcal{F}^{*}\right)$ admitting no unimodular elements, by Lemma 7.1 and Cor.7.2.5 of [2] we can and do identify $\mathcal{E}$ with $\mathcal{F} \oplus \mathcal{F}^{*}$. Let $\tilde{\psi}$ be an isometry of $\rho(\mathbf{q})$ with $\rho\left(\left(\begin{array}{ll}0 & 1 \\ \epsilon & 0\end{array}\right)\right)$. Then by [2], Cor.7.2.5 there exists an automorphism $\psi$ of $\mathcal{E}$ such that $H^{1}(\psi)=\tilde{\psi}$. By the definition of $\rho$ we have $\rho\left(\psi^{*} \mathbf{q} \psi\right)=\beta_{E} \operatorname{Ext}^{1}\left(\Gamma\left(\psi^{*}\right) q \Gamma(\psi)\right)$

$=\beta_{E} \operatorname{Ext}^{1}\left(\Gamma\left(\psi^{*}\right) \operatorname{Ext}^{1}(q) \operatorname{Ext}^{1}(\Gamma(\psi))\right.$, where $E=\Gamma(\mathcal{E})$ and $q=\Gamma(\mathbf{q})$. By $(1.2(2))$, we have $\beta_{E} \operatorname{Ext}^{1}\left(\Gamma\left(\psi^{*}\right)\right)=\operatorname{Ext}^{1}(\Gamma(\psi))^{\vee} \beta_{E}$, so that $\rho\left(\psi^{*} \mathbf{q} \psi\right)=$ $\operatorname{Ext}^{1}(\Gamma(\psi))^{\vee} \beta_{E} \operatorname{Ext}^{1}(q) \operatorname{Ext}^{1}(\Gamma(\psi))=\operatorname{Ext}^{1}(\Gamma(\psi))^{\vee} \rho(\mathbf{q}) \operatorname{Ext}^{1}(\Gamma(\psi))$

$=H^{1}(\psi)^{\vee} \rho(\mathbf{q}) H^{1}(\psi)=\tilde{\psi} \rho(\mathbf{q}) \tilde{\psi}$. We replace $\mathbf{q}$ by $\psi^{*} \mathbf{q} \psi$ and assume that $\rho(\mathbf{q})=\left(\begin{array}{ll}0 & 1 \\ \epsilon & 0\end{array}\right)$. Let $\mathbf{q}=\left(\begin{array}{cc}\alpha & \delta \\ \epsilon \delta^{*} & \beta\end{array}\right)$ with $\alpha: \mathcal{F} \rightarrow \mathcal{F}^{*}, \beta: \mathcal{F}^{*} \rightarrow \mathcal{F}$, $\delta: \mathcal{F}^{*} \rightarrow \mathcal{F}^{*}$ maps such that $\alpha^{*}=\epsilon \alpha, \beta^{*}=\epsilon \beta$. Since $\rho(\mathbf{q})=\left(\begin{array}{ll}0 & 1 \\ \epsilon & 0\end{array}\right)$, 
$H^{1}(\alpha)=0$ and $H^{1}(\beta)=0$. Therefore, by $\left([\mathrm{H}]\right.$, Cor.7.2.5), there exist $f_{1}$ : $\mathcal{F} \rightarrow \mathcal{O}_{Y}^{n}, f_{2}: \mathcal{F}^{*} \rightarrow \mathcal{O}_{Y}^{n}, g_{1}: \mathcal{O}_{Y}^{n} \rightarrow \mathcal{F}^{*}$ and $g_{2}: \mathcal{O}_{Y}^{n} \rightarrow \mathcal{F}$ such that $\alpha=g_{1} f_{1}$ and $\beta=g_{2} f_{2}$. Let us consider the automorphism

$$
\psi=\left(\begin{array}{cccc}
1 & 0 & -g_{1} / 2 & f_{1} * \\
0 & 1 & -g_{2} / 2 & f_{2}^{*} \\
0 & 0 & 1 & 0 \\
0 & 0 & 0 & 1
\end{array}\right)
$$

of $\mathcal{F} \oplus \mathcal{F}^{*} \oplus \mathcal{O}_{Y}{ }^{n} \oplus \mathcal{O}_{Y}{ }^{n *}$. We have

$$
\mathbf{q}^{\prime}=\psi\left(\begin{array}{cccc}
\alpha & \delta & 0 & 0 \\
\epsilon \delta^{*} & \beta & 0 & 0 \\
0 & 0 & 0 & 1 \\
0 & 0 & \epsilon & 0
\end{array}\right) \psi^{*}=\left(\begin{array}{cccc}
0 & X & \epsilon f_{1}^{*} & -g_{1} / 2 \\
\epsilon X^{*} & 0 & \epsilon f_{2}^{*} & -g_{2} / 2 \\
f_{1} & f_{2} & 0 & 1 \\
-\epsilon g_{1}^{*} / 2 & -\epsilon g_{2}^{*} / 2 & \epsilon & 0
\end{array}\right)
$$

where $X=\delta-\epsilon f_{1}^{*} g_{2}^{*} / 2-g_{1} f_{2} / 2$. Since $\Gamma(\mathcal{F})$ and $\Gamma\left(\mathcal{F}^{*}\right)$ admit no unimodular elements, $f_{1} \equiv f_{2} \equiv 0 \bmod m$. Hence $X$ is an isomorphism. Since $\mathbf{q}^{\prime}$ restricted to $\mathcal{F} \oplus \mathcal{F}^{*}$ is

$$
\left(\begin{array}{cc}
0 & X \\
\epsilon X^{*} & 0
\end{array}\right)
$$

with $X$ an isomorphism, $\mathbf{q}^{\prime}$ splits as

$$
\left(\begin{array}{cc}
0 & X \\
\epsilon X^{*} & 0
\end{array}\right) \perp \mathbf{q}^{\prime \prime}
$$

for some $\mathbf{q}^{\prime \prime}$ supported on a bundle $\mathcal{E}^{\prime \prime}$. Since $\mathcal{E} \oplus \mathcal{O}_{Y}{ }^{n} \oplus \mathcal{O}_{Y}{ }^{n *} \simeq \mathcal{E} \oplus \mathcal{E}^{\prime \prime}$, by $[2]$, Cor.7.2.5, $\mathcal{E}^{\prime \prime}$ is a trivial bundle and hence $\mathbf{q}^{\prime \prime}$ is in the image of $W^{\epsilon}(A)$. Since

$$
\mathbf{q} \perp H\left(\mathcal{O}_{Y}{ }^{n}\right) \simeq \mathbf{q}^{\prime} \simeq\left(\begin{array}{cc}
0 & X \\
\epsilon X^{*} & 0
\end{array}\right) \perp \mathbf{q}^{\prime \prime}
$$

it follows that $\mathbf{q}$ is in the image of $W^{\epsilon}(A)$.

Lemma 3.2 Let $M$ be a finite length $A$-module and $\psi: M \rightarrow M^{\vee}$ be an $\epsilon$-symmetric isomorphism. Then there exists a vector bundle $\mathcal{E}$ over $Y$ with a $(-\epsilon)$-symmetric isomorphism $\mathbf{q}: \mathcal{E} \rightarrow \mathcal{E}^{*}$ such that $\rho(\mathbf{q})=\psi$. 
Proof. Let $\mathcal{E}$ be a vector bundle on $Y$ such that $H^{1}(Y, \mathcal{E})=M(\operatorname{cf} \S 2)$. and $E=\Gamma(\mathcal{E})$ has no unimodular elements. Then $(\mathrm{cf} \S 2)$ the projective dimension of $E$ is less than or equal to 1 and $\operatorname{Ext}^{1}\left(E^{*}, A\right) \simeq M$. Let

$$
0 \rightarrow P_{1} \rightarrow P_{0} \rightarrow E \rightarrow 0
$$

and

$$
0 \rightarrow Q_{1} \rightarrow Q_{0} \rightarrow E^{*} \rightarrow 0
$$

be projective resolutions of $E$ and $E^{*}$ respectively. By dualising the projective resolution of $E^{*}$ we get an exact sequence

$$
0 \rightarrow E \rightarrow Q_{0}^{*} \rightarrow Q_{1}^{*} \rightarrow M \rightarrow 0 .
$$

By taking the Yoneda composition of this exact sequence with the projective resolution of $E$ we get a projective resolution

$$
0 \rightarrow P_{1} \rightarrow P_{0} \rightarrow Q_{0}^{*} \rightarrow Q_{1}^{*} \rightarrow M \rightarrow 0
$$

of $M$. By dualising this we get a projective resolution

$$
0 \rightarrow Q_{1} \rightarrow Q_{0} \rightarrow P_{0}^{*} \rightarrow P_{1}^{*} \rightarrow M^{\vee} \rightarrow 0
$$

of $M^{\vee}$. By lifting the $\epsilon$-symmetric isomorphism $\psi: M \rightarrow M^{\vee}$, we get a commutative diagram

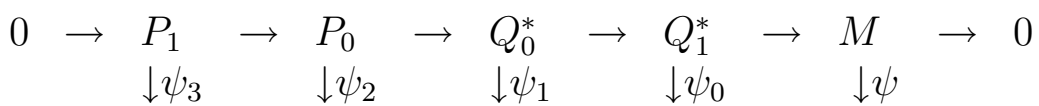

$$
\begin{aligned}
& 0 \rightarrow Q_{1} \rightarrow Q_{0} \rightarrow P_{0}^{*} \rightarrow P_{1}^{*} \rightarrow M^{\vee} \rightarrow 0
\end{aligned}
$$

of exact sequences. Since $E$ has no unimodular elements it is easy to see, as in the proof of (2.6), that the above diagram induces a $(-\epsilon)$-symmetric space $\mathbf{q}$ on $\mathcal{E}$ such that $\rho(\mathbf{q})=\psi$.

Theorem 3.3 Let $A$ be a regular local ring of dimension 3 and $m$ its maximal ideal. Assume that 2 is invertible in $A$. Let $Y=\operatorname{Spec}(A) \backslash\{m\}$. Then the the complex

$$
0 \rightarrow W^{\epsilon}(A) \stackrel{\iota}{\rightarrow} W^{\epsilon}(Y) \stackrel{\rho}{\rightarrow} W_{f l}^{-\epsilon}(A) \rightarrow 0
$$

is exact, where $\iota$ is induced by the restriction. 
Proof. If $\epsilon=1$, then the injectivity of $\iota$ follows from the injectivity of the canonical homomorphism $W(A) \rightarrow W(K)([\mathbf{6}]$, Th. 23), where $K$ is the quotient field of $A$. If $\epsilon=-1, \iota$ is injective because $W^{-1}(A)=0$.

We now prove the exactness in the middle. As we remarked in $\S 2, \rho \iota=0$. Let $(\mathcal{E}, \mathbf{q})$ be an $\epsilon$-symmetric space over $Y$ such that $\rho(\mathbf{q})$ is zero in $W_{f l}^{-\epsilon}(A)$. Then, by $(0.4), \rho(\mathbf{q})$ is metabolic. We show that $(\mathcal{E}, \mathbf{q})$ is in the image of $\iota$. In view of $(1.3)$, we assume that $\Gamma(\mathcal{E})$ has no unimodular elements. Then, by (2.6), there exist $\epsilon$-symmetric spaces $\mathbf{q}_{1}$ and $\mathbf{q}_{2}$ supported respectively on $\mathcal{E}$ and $\mathcal{O}_{Y}{ }^{n}$ for some integer $n$, such that $\mathbf{q}_{1} \perp \mathbf{q}_{2}$ is metabolic and $\rho(\mathbf{q}) \simeq \rho\left(\mathbf{q}_{1}\right)$. Thus $\rho\left(\mathbf{q} \perp-\mathbf{q}_{1}\right)$ is isomorphic to a hyperbolic space. Since $\Gamma(\mathcal{E} \oplus \mathcal{E})$ has no unimodular elements, it follows from (3.1) that $\mathbf{q} \perp-\mathbf{q}_{1}$ is in the image of $\iota$. Since $\mathbf{q}_{2}$ is in the image of $\iota$ and $\mathbf{q}_{1} \perp \mathbf{q}_{2}$ is metabolic, $\mathbf{q}_{1}$ and hence $\mathbf{q}$ is in the image of $\iota$.

The surjectivity of $\rho$ follows from (3.2).

Let $A$ be any regular ring. Let $\operatorname{Spec}^{1}(A)$ denote the set of prime ideals of $A$ of height 1 . Then for any $P \in \operatorname{Spec}^{1}(A)$, the local ring $A_{P}$ is a discrete valuation ring. Let $\partial_{P}: W(K) \rightarrow W\left(A_{P} / P A_{P}\right)$ denote the second residue homomorphism with respect to some choice of a parameter of $P A_{P}$, where $K$ is the quotient field of $A$.

Corollary 3.4 Let $A$ be a regular local ring of dimension $3, m$ its maximal ideal and $K$ its quotient field. Assume that 2 is invertible in $A$. The sequence

$$
0 \rightarrow W(A) \rightarrow W(K) \stackrel{\oplus \partial_{P}}{\rightarrow} \bigoplus_{P \in \operatorname{Spec}^{1}(A)} W\left(A_{P} / P A_{P}\right)
$$

is exact.

Proof. The injectivity of $W(A) \rightarrow W(K)$ is proved in [6], Th. 23. Since $W_{f l}^{-1}(A) \simeq W^{-1}(A / m)=0$, by (3.3) we have $W(A) \simeq W(Y)$. Thus it is enough to prove that the complex

$$
W(Y) \rightarrow W(K) \stackrel{\oplus \partial_{P}}{\longrightarrow} \bigoplus_{P \in \operatorname{Spec}^{1}(A)} W\left(A_{P} / P A_{P}\right)
$$

is exact. Let $q$ be a quadratic space over $K$ such that $\partial_{P}(q)=0$ for all height 1 prime ideals $P$ of $A$. Since $Y$ is a regular scheme of dimension 2 , by [1] 
2.5 p. 112 , there exists a quadratic space $(\mathcal{E}, \mathbf{q})$ over $Y=\operatorname{Spec}(A) \backslash\{m\}$, such that its image in $W(K)$ under the restriction map, is equal to $q$. This completes the proof of corollary.

Using (3.4), one can prove the following theorem (cf [8], Prop. 2.1).

Corollary 3.5 Let $A$ be a regular ring of dimension 3 and $K$ its quotient field. Assume that 2 is invertible in $A$. The sequence

$$
0 \rightarrow W(A) \rightarrow W(K) \stackrel{\oplus \partial_{P}}{\rightarrow} \underset{P \in \operatorname{Spec}^{1}(A)}{\bigoplus} W\left(A_{P} / P A_{P}\right)
$$

is exact.

We end this paper by giving a computation of $W^{-1}(Y)$ using (3.3).

Corollary 3.6 Let $A$ be a regular local ring of dimension 3 and $m$ its maximal ideal. Assume that 2 is invertible in $A$. Let $Y=\operatorname{Spec}(A) \backslash\{m\}$. Then $W^{-1}(Y) \simeq W(A / m)$.

Proof. Since $W^{-1}(A)=0$ and $W_{f l}(A) \simeq W(A / m)$, the result follows from (3.3). 


\section{References}

1. J.-L. Colliot-Thélène and J.-J. Sansuc: Fibrés quadratiques et composantes connexes réelles, Math. Ann. 244 (1979), 105-134.

2. G. Horrocks: Vector bundles on the punctured spectrum of a local ring, Proc. London Math. Soc., 14 (1964), 689-713.

3. M. Knebusch: Symmetric bilinear forms over algebraic varieties, Conference on quadratic forms, Kingston, Queen's papers in pure and applied math. 46 (1977), 103-283 .

4. S. Mac Lane : Homology, Grundlehren der Math. Wiss., 114, SpringerVerlag, Berlin-Heidelberg-New York, 1963.

5. H. Matsumura: Commutative ring theory, Cambridge stud. in adv. math. 8, Cambridge Univ. Press, 1986.

6. M. Ojanguren: A splitting theorem for quadratic forms, Comm. Math. Helv. 57 (1982), 145-157.

7. W. Pardon: A. Gersten conjecture for Witt groups, Lecture notes in Math. 967, Springer-Verlag, Berlin Heidelberg New York, 1982, 300315.

8. R. Parimala: Witt groups of affine three-folds, Duke Math. Jour. 57 (1988), 947-954.

9. W. Scharlau: Quadratic and Hermitian Forms, Grundlehren der Math. Wiss., 270, Springer-Verlag, 1985.

Université de Lausanne

Section de Mathématiques

CH-1015 Lausanne-Dorigny.

School of Mathematics

Tata Institute of Fundamental Research

Homi Bhabha Road

Bombay 400 005, India. 